\title{
Role of gut microbes in avian energy metabolism
}

\begin{abstract}
Gastrointestinal tract (GIT) of animals including birds house one of the most complex microbial ecosystems containing trillions of microbes. These microbes are distributed throughout various niches performing diverse functions including the fermentation of foods. The microbial diversity within the GIT is a function of site of digestion, health status and age of birds. Crop and ceca have the highest concentration of useful microbes like Lactobacillus. The fermentation of sugar and dietary fibre produces short chain fatty acids (SCFAs) which provide an efficient source of energy to the host and at the same time decreases the $\mathrm{pH}$ of the tract restricting the growth of harmful microorganisms. In commercial birds, however, gut microbes may not prove productive.
\end{abstract}

Keywords: gut microbes, birds, energy metabolism, crops, mammals
Volume 3 Issue 2 - 2018

\author{
Shahid Hassan Mir,' Shabir Ahmed Lone ${ }^{2}$ \\ 'Animal Nutrition Division, India \\ ${ }^{2}$ ICAR-National Dairy Research Institute, India
}

Correspondence: Shabir Ahmed Lone, Animal Reproduction Gynaecology and Obstetrics, ICAR-National Dairy Research Institute (NDRI), Karnal, Haryana-I3200I, India, Email drloneshabir@gmail.com

Received: January 28, 2018 | Published: March 14, 2018

\section{Introduction}

Microorganisms are universally present in the different ecosystems, performing diverse functions. One of the important places is the gastrointestinal tract of animals. Some of the microbes in the gut are useful while some are not so useful and some are even dangerous or diseases causing. Microbes are normally present in the gut such that their disease-inducing activity (dysbiosis) and disease-protective activity (probiosis) is perfectly balanced. The symbiotic relationship between the host and the gut-microbes has long been established. This relationship can explicitly be visualized in ruminants where they depend on gut microbes for life. Scientists trace back the origin of this symbiotic relationship to 15 million years. It is believed that organisms co-evolve with their gut microbiota and eventually develop a symbiotic relationship with them to achieve a physiological homeostasis. ${ }^{1}$ The symbiosis between the host and gut microbiota can trigger specific biological responses, both locally and systemically which can prove beneficial to both of them of the 55 bacterial divisions, only two are prominent in mammals and birds (Bacteroidetes and Firmicutes). ${ }^{2}$ The beneficial effects of gut microbes include food digestion thus seconding the energy metabolism. Disturbed microbial ecology can impair many of the homeostatic and physiological signals which may result in a number of diseases.

Birds have the specially designed gastrointestinal tract which helps them in flight. Energy metabolism in birds is largely controlled by enzymes, however, gut-microbes inhabiting mostly in the large intestine have a significant effect on the energy metabolism. In case of birds, fermentation of sugar and fibre mostly occur in the crop and ceca, however, it depends on the species of bird, captive type and the diet. ${ }^{3}$ The introduction of the avian gut microbes and their diversity has been reviewed by Roto ${ }^{4}$ and Kohl ${ }^{5}$ respectively. The gut microbiome significantly increases the dietary energy (ME) in birds, indicating that the microbiota are responsible for extracting additional dietary energy from the feed. The products of fermentation like volatile fatty acids (VFAs) are not utilized to the extent of ruminants. Moreover, the energy obtained from microbial biomass in ruminants is not obtained in birds, although some birds do coprophagy as an adaptive strategy to combat nutrient deficiency.

\section{Esophagus, crop and Stomach}

The environment of a crop (ingluvies) favours the microbial growth by providing optimum temperature of $40^{\circ} \mathrm{C}$ with favouable anaerobic conditions. The microbiota in the crop of birds changes with age and diet. The crop and lower esophagus mostly inhabit the bacteria belonging to Lactobacillus spp. especially Lactobacillus salivarius, $L$. fermentum, L. reuteri and $L$. acidophilus. These microbes are mostly attached to the wall of the crop. The colonization of these bacteria occurs in just one hour after hatching. The concentration of bacteria in the crop is about $10^{9} / \mathrm{g} .{ }^{6}$ Birds like Hoatzin and Kakapo have large crops and their microbial composition closely relates to that of the pre-gastric fermentors. There is an appreciable fermentation of starch in the crop producing short chain fatty acids (SCFAs) which decreases its $\mathrm{pH}$. Starch is degraded by microbial amylase to produce maltose, maltotriose and glucose. These sugars may be absorbed by the mucous membrane of the crop or used as a substrate for the production of volatile fatty acids. Some fibre degrading activities of cellulolytic bacteria have also been found in the crop, but it is inappreciable except in Hoatzin. The food stored in the crop contributes about $75.5 \%$ of nocturnal energy requirements.

The proventriculus and gizzard together forms the stomach of the birds. It is the site for the enzymatic digestion. The lower $\mathrm{pH}$ makes the presence of microbes difficult; however, some resisitant bacteria can be found attached to the walls, although they don't contribute to any fermentation processes except in birds like ostriches (total anaerobes $3 \times 10^{6} / \mathrm{g}$ ingesta) where the uneven mixing of digesta might produce pockets of high $\mathrm{pH}$ producing favourable conditions for the growth of microbes. ${ }^{7}$

\section{Intestine}

Birds possess relatively short intestine, however, the length of the intestine can be increased (as much as $40 \%$ ) in some species by switched to the high-fiber diet. Terminal restriction fragment length polymorphism (T-RFLP) analysis indicates that different sections of intestines harbor different microbial communities except for duodenum and jejunum. Moreover, diet doesn't seem to affect the composition of microbial communities of duodenum and jejunum. ${ }^{8}$ Cultivation-based techniques reveal that most of the intestinal microbes are Gram-positive rods (77\%), followed by Gram-negative rods (14\%) and Gram-positive cocci (9\%). These bacteria include Eubacterium, Lactobacillus, Peptostreptococcus, Escherichia coli, Propionibacterium, and Bacteroides. Lactobacilli predominate in the duodenum of chickens. ${ }^{9}$ The relationship between the intestinal microbiota and the host nutritional metabolism is poorly understood. Some amount of the fermentation occurs in the proximal part of the 
small intestine; while appreciable amount of fermentation occurs in distal part of intestines like ileum. In some birds like emu and geese, the ileum is of sufficient length to retain the digesta long enough for fermentation to occur, resulting in the digestion of some components of plant cell walls like hemicelluloses $(45 \%)$ and cellulose $(19 \%) .{ }^{10}$

\section{Ceca}

The ceca of birds harbor complex microbiome which largely consists of the bacterial population. Studies by culture-dependent techniques revealed a high abundance $\left(10^{10}-10^{11} / \mathrm{g}\right.$ microscope counts) of anaerobic bacteria in the cecum. These include anaerobic Gram-negative cocci, facultative anaerobic cocci, and streptococci. Peptostreptococcus, Propionibacterium, Eubacterium, Bacteroides, and Clostridium are the major genera identified from the cecum. Only $20-60 \%$ of the total cecal bacteria could be cultivated in the media. Now, over 200 different bacteria have been isolated which can be divided into three types viz: dominating, sub-dominating, and temporary. ${ }^{11}$ The predominant cecal bacteria on the first days of life are Enterobacteriacae spp., Enterococus spp. and Lactobacillus spp., while Bacteroides spp. and Eubacterium spp. are established after 12 days. ${ }^{12}$ Lactobacillus acidophilus, Lactobacillus salivarius, and Lactobacillus fermentum are the important Lactobacillus bacteria which appears in significant numbers $\left(10^{8}-10^{10} \mathrm{~g}^{-1}\right)$ by the end of the first week. ${ }^{13}$ Later, large populations of non-spore forming anaerobes has been isolated. Lack of intimate contact with the dam may cause delayed development of cecal microflora as maternal grooming and oral aerosols are important ways of inoculating young animals. The diversity of cecal microbes helps them to utilize different substrates like Glucose, Lactose, Lactate, Ammonia and Uric acid. The cecal microflora can also utilize some amount of fibre which is not digested by the host enzymes. The short chain fatty acids including butyrate, acetate, lactate, propionate, valerate, and isovalerate are produced during cecal fermentation. ${ }^{14}$ Glucose is an important energy source in birds; however, SCFAs may also be used as an energy source. ${ }^{15}$ Some birds like ostrich and emu can obtain as much as $75 \%$ and $50 \%$, respectively, of their maintenance energy requirement from the fermentation process which is comparable to ruminants. ${ }^{16}$ Some of the fermentation activities are also reported in the colon of birds. Although SCFAs provide a potential source of energy to the host, however, only a small proportion $(25 \%)$ is actually utilized by the bird.

Although birds have a crop, two ceca and a colon for fermentation of food residues, the commercial birds are believed to gain little or nothing from fermentation as they are fed solely on the concentrate diets. Interestingly, some scientists suggest that the gastrointestinal microbiome are more of a disadvantage than an advantage as 'germ free' birds tend to grow larger than normal birds. Moreover, SCFAs, which are regarded as the high metabolic energy value compounds, are inefficiently utilized by the host. Hence, the levels of SCFAs do not reflect the net deposition of energy by the host. Gut microbiota are also believed to increase the cost of energy available to host by altering the rate of energy-consuming reactions.

\section{Conclusion}

Gut microbes form an integral part of the energy metabolism of the host. They tend to utilize those compounds which otherwise is not digested by the host and produce high energy compounds like SCFAs thereby increasing the metabolizable energy of the feed. However, in commercial birds gut microbes might prove a disadvantage as they tend to hamper the utilization of high energy feed.

\section{Acknowledgements}

None.

\section{Conflict of interest}

Authors declares there is no conflict of interest.

\section{References}

1. Moeller AH, Caro Quintero A, Mjungu D, et al. Cospeciation of gut microbiota with hominids. Science. 2016;353(6297):380-382.

2. Waite DW, Taylor M. Exploring the avian gut microbiota: current trends and future directions. Front Microbiol. 2015;6(3):673.

3. Waite DW, Taylor MW. Characterizing the avian gut microbiota: membership, driving influences, and potential function. Front Microbiol. 2014;(5):223.

4. Roto SM, Rubinelli PM, Ricke SC. An introduction to the avian gut microbiota and the effects of yeast-based prebiotic-type compounds as potential feed additives. Front Vet Sci. 2015;(2):28.

5. Kohl KD. Diversity and function of the avian gut microbiota. $J$ Comp Physiol B. 2012;182:591-602.

6. Oakley BB, Lillehoj HS, Kogut MH, et al. The chicken gastrointestinal microbiome. FEMS microbiology letters. 2014;(360):100-112.

7. Swart D, Mackie RI, Hayes JP. Influence of live mass, rate of passage and site of digestion on energy metabolism and fibre digestion in the ostrich (Struthio camelus var. domesticus). South African Journal of Animal Science. 1993;(23):119-126.

8. Yan W, Sun C, Yuan J, et al. Gut metagenomic analysis reveals prominent roles of Lactobacillus and cecal microbiota in chicken feed efficiency. Scientific reports. 2017;(7):45308.

9. Ouwehand AC, Vaughan EE, editors. Gastrointestinal microbiology. CRC Press: USA; 2006.

10. Buchsbaum R, Wilson J, Valiela I. Digestibility of plant constituents by Canada geese and Atlantic brant. Ecology. 1986;67(2):386-393.

11. Barnes EM. The intestinal microflora of poultry and game birds during life and after storage. Journal of Applied Microbiology. 1979;(46):407419.

12. Van der Wielen PW, Biesterveld S, Lipman LJ, et al. Inhibition of a glucose-limited sequencing fed-batch culture of Salmonella enterica serovar Enteritidis by volatile fatty acids representative of the ceca of broiler chickens. Appl Environ Microbiol. 2001;67(4):1979-1982.

13. Mead GC. Microbes of the avian cecum: types present and substrates utilized. Journal of Experimental Zoology Part A: Ecological Genetics and Physiology. 1989;252:48-54.

14. Jamroz D, Wiliczkiewicz A, Skorupinska J, et al. Fermentation and apparent digestion of the structural carbohydrates in chicks, ducks and geese fed triticale mixtures supplemented with enzyme. Journal of Animal Physiology and Animal Nutrition (Germany). 1998;(79):1-17.

15. Kirchgessner VM, Eder K, Müller HL, et al. Zur energetischen Bewertung von Nichtstärke Polysacchariden beim Geflügel. Journal of Animal Physiology and Animal Nutrition. 1999;(81):51-56.

16. Herd RM, Dawson TJ. Fiber digestion in the emu, Dromaius novaehollandiae, a large bird with a simple gut and high rates of passage. Physiological Zoology. 1984;(57):70-84. 